\title{
Criterios asumidos por profesores cuando argumentan sobre la creatividad matemática'
}

\author{
María José Seckel ${ }^{2}$ \\ ORCID: 0000-0001-7960-746X \\ Adriana Breda ${ }^{3}$ \\ ORCID: 0000-0002-7764-0511 \\ Alicia Sánchez ${ }^{3}$ \\ ORCID: 0000-0001-6569-6828 \\ Vicenç Font ${ }^{3}$ \\ ORCID: 0000-0003-1405-0458
}

\section{Resumen}

El propósito de este estudio fue identificar cuáles son los criterios asumidos por profesores en ejercicio cuando argumentan sobre el desarrollo de la creatividad en los procesos de enseñanza y aprendizaje de las matemáticas. Para ello, se analizó el discurso de 35 profesores estudiantes de másters en Educación Matemática de tres universidades chilenas. Los datos, con enfoque metodológico mixto, se recogieron a partir de un cuestionario de preguntas cerradas y abiertas. Las preguntas cerradas se enfocaron en cuatro ámbitos: 1) creatividad matemática y procesos creativos; 2) creatividad en el ámbito profesional; 3) creatividad en las matemáticas; y 4) promoción de la creatividad en la enseñanza y aprendizaje de la matemática. Para el análisis de preguntas abiertas, donde los profesores presentaron un ejemplo de actividad matemática creativa y destacaron lo creativo de ella, se consideraron como categorías de análisis los Criterios de Idoneidad Didáctica (CI) propuestos en el Enfoque Ontosemiótico de la Instrucción y Cognición matemática (EOS). Los resultados muestran, entre otros aspectos, que los profesores tienen una visión dual respecto a la creatividad matemática (por una parte, es innata $\mathrm{y}$, por otra parte, puede ser enseñada) y que, cuando argumentan sobre ella, utilizan de manera implícita criterios epistémicos, mediacionales, emocionales y ecológicos.

\section{Palabras clave}

Creatividad matemática - Criterios de idoneidad - Profesores de matemática.

1- Trabajo desarrollado en el marco de los proyectos de investigación en formación de profesorado: PGC2018-098603-B-100 (MCIU/AEI/FEDER, UE), REDICE18-2000 (ICE-UB) y 434218 (Desarrollo de la competencia reflexiva en el contexto de prácticas progresivas de futuros profesores Educación General Básica con Mención en Matemática, Chile).

2- Universidad Católica del Maule, Maule, Chile. Contacto: mjseckel@ucm.cl.

3- Universidad de Barcelona, Barcelona, España. Contactos: adriana.breda@gmail.com, asanchezb@ub.edu, vfont@ub.edu. 


\section{Criteria assumed by teachers when they argue about mathematical creativity}

\section{Abstract}

The aim of this study was to identify the criteria assumed by in-service teachers when they argue about the development of creativity in the mathematical teaching and learning processes. To do so, we analyzed the discourse of 35 teachers who were taking master's degrees in Mathematics Education in three Chilean universities. Using a mixed methodological approach, data was collected through a questionnaire including closed and open questions. The closed questions focused on four topics: 1) mathematical creativity and creative processes; 2) creativity in the professional environment; 3) creativity in mathematics; and 4) enhancement of creativity in teaching and learning of mathematics. To analyze the open questions, whose purpose was that the teachers presented an example of creative mathematical activity and highlighted which of its characteristics made the activity creative, we considered the Didactic Suitability Criteria (DSC) proposed by the Onto-semiotic Approach (OSA) to Mathematical Instruction and Cognition as categories of analysis. Results show, among other aspects, that the teachers have a dual view of mathematical creativity (on the one hand, it is innate and, on the other hand, it can be taught) and they implicitly use epistemic, mediational, emotional, and ecological criteria when arguing about mathematical creativity.

\section{Keywords}

Mathematical creativity - Suitability criteria - Mathematics teachers.

\section{Introducción}

La creatividad es una temática relativamente nueva en el ámbito de la investigación, que toma interés de manera especial a mediados del siglo XX (MALLART; DEULOFEU, 2017). Hasta la fecha diversos autores han intentado identificar los rasgos principales de la creatividad (VIOLANT, 2006; MARÍN; DE LA TORRE, 1991; LOGAN; LOGAN; GARCÍA, 1980) y hay un cierto consenso en que la creatividad conlleva al menos tres aspectos: 1) fluidez; 2) flexibilidad; y 3) originalidad. Por otra parte, en el World Economic Forum, la creatividad fue presentada como una de las competencias a desarrollar en la educación del siglo XXI, junto con el pensamiento crítico, comunicación y colaboración (CORFO; FUNDACIÓN CHILE, 2017).

En las investigaciones que han abordado el tema de la creatividad matemática (REYES, 2003; BARQUERO et al., 2014) se observa que el concepto es un término polisémico en el que, según Silver (1997) y Sriraman (2009), coexisten dos visiones. La primera es la visión de la creatividad del genio, que sugiere que la creatividad matemática difícilmente puede ser modificada con procesos formativos y que el trabajo creativo surge 
de momentos de inspiración puntual que pocos consiguen y, la segunda, es la visión de creatividad que se asocia a largos procesos de estudio y de indagación que, a través de procesos de formación adecuados, puede llegarse a desarrollar.

Algunos autores destacan que el desarrollo de la creatividad matemática significa una alternativa de cambio en el campo educacional, necesario para enfrentar los desafíos de la vida actual (REYES, 2003), sin embargo, bajo la segunda visión que se presenta en este apartado, se observa que los profesores no implementan estrategias de enseñanza que permitan su desarrollo, por tanto, es necesario presentar la creatividad a los profesores y futuros profesores como parte de su formación (SRIRAMAN, 2005).

En este contexto surge la presente investigación, cuyo propósito es identificar los criterios asumidos por profesores cuando argumentan sobre creatividad en los procesos de enseñanza y aprendizaje de las matemáticas.

\section{Marco teórico}

\section{Los criterios de idoneidad didáctica como organizadores del discurso del profesor sobre la creatividad matemática.}

La noción de idoneidad didáctica propuesta en el Enfoque Ontosemiótico (EOS a partir de ahora) (BREDA; FONT; LIMA, 2015) es una respuesta parcial a la siguiente problemática: ¿Qué criterios se deben utilizar para diseñar una secuencia de tareas, que permitan evaluar y desarrollar la competencia matemática de los alumnos y qué cambios se deben realizar en su rediseño para mejorar el desarrollo de esta competencia? Los criterios de idoneidad son útiles en dos momentos de los procesos de instrucción. A priori, los criterios de idoneidad son principios que orientan cómo se deben hacer las cosas. A posteriori, los criterios sirven para valorar el proceso de instrucción efectivamente implementado. En el EOS se consideran los siguientes criterios de idoneidad didáctica (FONT; PLANAS; GODINO, 2010): 1) Idoneidad Epistémica, para valorar si las matemáticas que están siendo enseñadas son buenas matemáticas; 2) Idoneidad Cognitiva, para valorar, antes de iniciar el proceso de instrucción, si lo que se quiere enseñar está a una distancia razonable de aquello que los alumnos saben, y después del proceso, si los aprendizajes adquiridos están cerca de aquello que se pretendía enseñar; 3) Idoneidad Interaccional, para valorar si las interacciones resuelven dudas y dificultades de los alumnos; 4) Idoneidad Mediacional, para valorar la adecuación de los recursos materiales y temporales utilizados en el proceso de instrucción; 5) Idoneidad Emocional, para valorar la implicación (intereses, motivaciones, etc.) de los alumnos durante el proceso de instrucción; 6) Idoneidad Ecológica, para valorar la adecuación del proceso de instrucción al proyecto educativo del centro, las directrices curriculares, las condiciones del entorno social y profesional.

La operatividad de los criterios de idoneidad (CI) exige definir un conjunto de indicadores observables, que permitan valorar el grado de idoneidad de cada uno de los criterios. En Breda y Lima (2016), Seckel (2016) y Breda, Pino-Fan y Font (2017) se aporta un sistema de indicadores, agrupados en componentes, que sirve de guía de análisis y valoración de la idoneidad didáctica, que está pensado para un proceso de instrucción en cualquier etapa educativa. 
Sánchez y Font (2017), en un contexto institucional donde se explican los criterios de idoneidad como una pauta para organizar la reflexión del futuro profesor de matemáticas de secundaria, estudian cómo aparece tratada la creatividad en los Trabajos de Fin de Máster de estos futuros profesores y, dado que la reflexión sobre la propia práctica de los futuros profesores se organiza a partir de los CI, estos autores también usan los CI para categorizar y clasificar los comentarios sobre la creatividad que hacen los futuros profesores. En particular, llegan a la conclusión de que en dichos comentarios la creatividad se asocia a diferentes aspectos, los cuales a su vez se relacionan con algún indicador o componente de los CI. Por ejemplo, la creatividad se asocia con proponer problemas abiertos (idoneidad epistémica); con la interacción de los estudiantes en situaciones de aprendizaje cooperativo (idoneidad interaccional); con la presentación de tareas motivadoras (idoneidad emocional), el uso de recursos tecnológicos y manipulativos (idoneidad de medios); el desarrollo de la ciudadanía y el pensamiento crítico (idoneidad ecológica) e incluso con la evaluación del aprendizaje (idoneidad cognitiva). Los análisis realizados en esta investigación muestran la gran variedad de aspectos con los que se relaciona la creatividad.

Por otra parte, en diversas investigaciones se ha observado el siguiente fenómeno: los criterios de idoneidad didáctica propuestos por el EOS funcionan como regularidades en el discurso de los profesores cuando justifican que sus propuestas didácticas representan una mejora, sin habérseles enseñado el uso de esta herramienta para guiar su reflexión. Por ejemplo, en Breda (2016) y Breda (en prensa) se presenta una investigación cuyo objetivo es comprender cómo los profesores justifican que sus propuestas didácticas representan una mejora de la enseñanza de las matemáticas. Al analizar el discurso de los profesores para justificar la calidad de su innovación, se observó un uso implícito de algunos de los componentes y descriptores de los diferentes criterios de idoneidad didáctica propuestos por el EOS, a pesar de que dicho constructo no era conocido por los profesores participantes - se trata de un fenómeno observado en otras investigaciones realizadas en diferentes países, por ejemplo (RAMOS, 2006; SECKEL, 2016; MORALES; FONT, 2017).

El motivo por el cual los criterios de idoneidad didáctica funcionan como regularidades en el discurso de los profesores, cuando estos tienen que justificar que sus propuestas representan una mejora, está relacionado con su origen consensual (BREDA; FONT; PINOFAN, 2018), ya que estos criterios, sus componentes e indicadores se han seleccionado a partir de la condición de que debían de contar con un cierto consenso en el área de Didáctica de las Matemáticas, aunque fuese local. Por tanto, una explicación plausible de que los criterios, sus componentes e indicadores funcionen como regularidades en el discurso del profesor es que reflejan consensos sobre cómo debe ser una buena enseñanza de las matemáticas ampliamente asumidos en la comunidad de educadores matemáticos; y es plausible pensar que el uso implícito que hace el profesor de ellos se debe a su formación y experiencia previa, la cual le hace partícipe de dichos consensos. Ahora bien, otra explicación también plausible es que el profesor que utiliza estos criterios, al no haber participado en el proceso de generación de los consensos que los soportan, los asuma como regularidades en su discurso simplemente porque se le presentan como algo naturalizado e incuestionable. Esta última explicación donde más plausible parece es en 
la formación de futuros profesores, ya que es evidente que ellos no han participado en la generación de los consensos que son el soporte de los criterios de idoneidad didáctica.

En esta investigación partimos del supuesto de que al preguntar sobre la incorporación de la creatividad en los procesos de enseñanza y aprendizaje de las matemáticas a futuros profesores que no conocen el constructo criterios de idoneidad didáctica, en cierta manera les estamos poniendo en situación de reflexionar sobre una posible mejora de la enseñanza de las matemáticas y esperamos que sus comentarios se puedan organizar también mediante los componentes e indicadores de los CI.

\section{Creatividad matemática}

La creatividad y, en particular, la creatividad matemática es un concepto complejo y polémico que ha sido considerado en muchas investigaciones y abordado desde muchos puntos de vista. Diversas caracterizaciones sobre qué es la creatividad, la creatividad en matemáticas y el Pensamiento Matemático Creativo (PMC) han sido propuestas, estando algunas de ellas centradas más en los sujetos creativos, en el proceso creativo o en los productos resultantes (HAYLOCK, 1987; MANN, 2006; SRIRAMAN, 2009; MALLART; DEULOFEU, 2017).

Entre otros trabajos destacados, encontramos Ervynck (1991) que describe la creatividad matemática a través de tres etapas: una etapa técnica conceptual preliminar, seguida de una actividad más algorítmica $y$, finalmente, una actividad creativa que consiste en la habilidad de resolver problemas y de tomar decisiones con estrategias no algorítmicas. Por su parte, Hadamard (1945) después de indagar en los procesos mentales que matemáticos y científicos seguían al hacer matemáticas, describe el proceso creativo utilizando el modelo Gestalt con sus cuatro fases: preparación-incubación-iluminaciónverificación. Trabajos más recientes, como el de Liljedahl (2013), aceptan y extienden los trabajos de Hadamard añadiendo el fenómeno que denomina AHA! experience que, según el autor, es clave en el momento de la iluminación. A pesar de la descripción más a menos aceptada de las etapas en los procesos creativos en matemáticas, no deja de ser misterioso el cómo promover los mecanismos adecuados para que la vivencia y transición de estos distintos momentos puedan ocurrir. En este sentido, los misteriosos mecanismos mediante los cuales actúa la incubación de los problemas con los que se ocupa la mente hasta el momento en que en ella surge la inspiración o iluminación decisiva en todo proceso verdaderamente creativo es otro de los temas poco conocidos y bastante controvertidos de los que habrá que ocuparse (GUZMÁN, 1995).

Algunos investigadores distinguen entre la creatividad matemática en la comunidad matemática y la creatividad en la enseñanza y aprendizaje de las matemáticas a nivel escolar. Por ejemplo, Kaufman y Beghetto (2008) distinguen entre big-C, middle-C o little-C para centrarse en la comunidad de matemáticos como productores de conocimiento, en los alumnos en grupo o en los alumnos individualmente. En este trabajo, nos centraremos en la creatividad a nivel escolar (o middle-C), que Liljedahl y Sriraman (2006) sugieren caracterizar como: "La creatividad matemática a nivel escolar es: (1) el proceso que da como resultado soluciones inusuales (nuevas) y/o perspicaces a un problema dado o problema análogo, y/o (2) la formulación de nuevas preguntas” (p. 19). 


\section{Diseño de la investigación}

El trabajo de investigación sigue una metodología mixta, con aspectos cuantitativos y cualitativos. Se aplicó un cuestionario como instrumento de recolección de datos con preguntas cerradas y abiertas. A través del apartado de preguntas abiertas se buscó profundizar las respuestas entregadas por los participantes en el apartado de preguntas cerradas. Dicho instrumento consideró un total de veintisiete reactivos donde tenían que indicar el grado de acuerdo (en una escala tipo Likert de 1 a 5), respecto a afirmaciones que se relacionaban con cuatro ámbitos de interés: 1) la creatividad matemática y los procesos creativos; 2) la creatividad en su ámbito profesional; 3) la creatividad en las matemáticas; 4) promoción de la creatividad matemática en la enseñanza y aprendizaje de la matemática. El instrumento había sido validado previamente en el proyecto A Computational Environment to Stimulate and Enhance Creative Designs for Mathematical Creativity (M C Squared) de la Unión Europea sobre el desarrollo de la creatividad matemática (BARQUERO et al., 2014), y dado que la población comparte condiciones semejantes a las originales en las que se desarrolló el instrumento, este no recibió adaptaciones (MUÑIZ, 2017). Dicho instrumento se aplicó a 35 profesores estudiantes de máster en Educación Matemática de tres universidades del sur de Chile, cuyos programas de postgrado tienen una duración de dos años académicos. Cabe destacar que, al momento de aplicar el instrumento, todos los participantes se encontraban cursando el último semestre de formación.

El análisis de los datos consideró, en una primera fase, técnicas descriptivas para el análisis de las preguntas cerradas del cuestionario a partir de porcentajes. En una segunda fase, se analiza el discurso que emana a partir de las cinco preguntas abiertas del instrumento con una técnica de análisis de contenido similar a la realizada en Breda (2016), donde se busca encontrar un texto que se pueda considerar una evidencia del uso implícito de algún indicador o componente de algún criterio de idoneidad didáctica (categorización deductiva), es decir, en esta fase del análisis, se toman los Criterios de Idoneidad Didáctica como categorías previas para analizar el discurso de los profesores. Por ejemplo, un párrafo como el siguiente: Que los alumnos creen sus situaciones problemas y las expliquen y justifiquen (participante C), se considera una evidencia del uso implícito del indicador riqueza de procesos uno de los componentes de la idoneidad epistémica. Ahora bien, para realizar este tipo de categorización, en el caso de las cuatro primeras preguntas abiertas, se llevó a cabo un paso previo en el que se levantaron categorías de tipo inductivas, con la finalidad de reconocer los tipos de elementos que evidencian la creatividad matemática y las actividades y acciones que la promueven (según los participantes). Asimismo, se da a conocer la frecuencia de aparición de las categorías, considerando el criterio de cuantificar la cantidad de participantes que evidenciaron fragmentos de discurso relacionados con estas.

Finalmente, en una tercera fase de análisis, se triangula la información de las fases anteriores con el propósito de establecer las ideas núcleo que surgen de los datos obtenidos.

\section{Resultados}

Los resultados obtenidos serán expuestos considerando el carácter mixto del instrumento implementado. En primer lugar se muestran los resultados correspondientes 
a la valoración que otorgan los participantes respecto de las afirmaciones presentadas en los cuatro ámbitos de interés y, posteriormente, se presenta la relación con los CI que emana de las preguntas abiertas del instrumento.

En la tabla 1 se observan las afirmaciones presentadas en cada ámbito. En ella se registra la frecuencia relativa porcentual en cada nivel de la escala de Likert, donde 1 corresponde a muy en desacuerdo, 2 a en desacuerdo, 3 a ni de acuerdo ni en desacuerdo, 4 a de acuerdo y 5 a completamente de acuerdo.

Tabla 1- Aspectos sobre la creatividad matemática

\begin{tabular}{|c|c|c|c|c|c|}
\hline Ámbito 1: Creatividad matemática y procesos creativos & $1(\%)$ & $2(\%)$ & $3(\%)$ & $4(\%)$ & $5(\%)$ \\
\hline 1. La creatividad es una cualidad o capacidad innata. & 9 & 9 & 28 & 31 & 23 \\
\hline 2. La creatividad es una cualidad que se puede desarrollar, educar, instruir, etc. & 2 & 3 & 8 & 37 & 50 \\
\hline 3. El pensamiento creativo es una consecuencia de momentos puntuales de inspiración. & 14 & 11 & 32 & 29 & 14 \\
\hline 4. El pensamiento creativo está asociado a un proceso de estudio largo y profundo de una situación problemática. & 17 & 20 & 34 & 17 & 12 \\
\hline 5. La interacción con otras personas y/o diferentes puntos de vista es importante para desarrollar un trabajo creativo. & 0 & 2 & 3 & 29 & 66 \\
\hline $\begin{array}{l}\text { 6. Para poder avanzar en un proceso creativo hay una formación rica y robusta en conocimientos específicos (en } \\
\text { matemáticas, en arte, en tecnología, etc.). }\end{array}$ & 8 & 8 & 20 & 29 & 35 \\
\hline 7. La interacción entre diferentes disciplinas no es relevante para generar creatividad. & 51 & 37 & 9 & 3 & 0 \\
\hline 8. En un proceso creativo siempre aparecen contribuciones originales o novedosas. & 17 & 20 & 6 & 26 & 31 \\
\hline 9. El proceso creativo suele priorizar una forma de abordar el/los problema/s. & 14 & 20 & 17 & 23 & 26 \\
\hline \multicolumn{6}{|l|}{ Ámbito 2: La creatividad en su ámbito profesional } \\
\hline 10. ¿En tu trabajo, o ámbito profesional, te consideras creativo/a? & 0 & 11 & 49 & 31 & 9 \\
\hline 11. Las matemáticas están presentes en las actividades profesionales. & 0 & 0 & 6 & 34 & 60 \\
\hline \multicolumn{6}{|l|}{ Ámbito 3: La creatividad en las matemáticas } \\
\hline 12. La matemática es una disciplina que ayuda a promover la creatividad en otras ramas del conocimiento. & 0 & 3 & 6 & 40 & 51 \\
\hline 13. Las matemáticas requieren más creatividad que el arte y la arquitectura. & 20 & 14 & 26 & 29 & 11 \\
\hline 14. Las matemáticas requieren menos creatividad que la ingeniería. & 26 & 23 & 31 & 17 & 3 \\
\hline \multicolumn{6}{|l|}{ Ámbito 4: Promoción de la creatividad en la enseñanza y aprendizaje de la matemática } \\
\hline \multicolumn{6}{|l|}{ (4A) ¿Cómo es un estudiante creativo? } \\
\hline 15. Es capaz de formular cuestiones e iniciar investigaciones. & 3 & 0 & 6 & 40 & 51 \\
\hline 16. Tiene un fuerte conocimiento de conceptos y herramientas matemáticas. & 9 & 11 & 37 & 34 & 9 \\
\hline 17. Sabe encontrar diferentes maneras de representar los conceptos o de llegar a la solución de un problema. & 0 & 0 & 11 & 26 & 63 \\
\hline \multicolumn{6}{|l|}{ (4B) ¿Cómo es un maestro o profesor creativo? } \\
\hline 18. Tiene una actitud transgresora hacia la enseñanza tradicional de las matemáticas. & 6 & 3 & 11 & 29 & 51 \\
\hline 19. Tiene un fuerte conocimiento de conceptos y herramientas matemáticas. & 5 & 0 & 26 & 20 & 49 \\
\hline 20. Tiene herramientas y recursos para estimular la creatividad en sus estudiantes. & 0 & 0 & 3 & 23 & 74 \\
\hline 21. Sabe cómo valorar y apoyar la creatividad en sus estudiantes. & 0 & 0 & 3 & 17 & 80 \\
\hline \multicolumn{6}{|l|}{ (4C) ¿Qué elementos consideras que tienen más o menos impacto en promover la creatividad matemática en nuestras aulas? } \\
\hline 22. La actitud del maestro o el profesor. & 0 & 0 & 3 & 11 & 86 \\
\hline 23. El uso de nuevas tecnologías para la enseñanza. & 3 & 11 & 26 & 34 & 26 \\
\hline 24. El quiebre disciplinar (que las matemáticas entren en contacto con otras disciplinas). & 0 & 3 & 6 & 31 & 60 \\
\hline 25. Un buen diseño de actividades matemáticas. & 0 & 0 & 3 & 17 & 80 \\
\hline 26. La/s actitud/es de los estudiante/s. & 0 & 0 & 11 & 32 & 57 \\
\hline 27. El contacto docencia con investigación. & 3 & 6 & 17 & 40 & 34 \\
\hline
\end{tabular}

Fuente: elaboración propia. 
En el ámbito 1, respecto a la creatividad matemática, se puede observar que un $50 \%$ de los participantes señala estar completamente de acuerdo en que esta es una capacidad que se puede desarrollar e instruir, frente a un 23\% que manifiesta que dicha capacidad es innata. En cuanto a los procesos creativos se destaca la alta valoración que otorgan los participantes (66\%) a la interacción con otras personas y la interacción entre disciplinas para generar creatividad (51\%). En el ámbito 2 se observa que un bajo porcentaje se considera creativo en su trabajo (9\%), mientras que un 60\% señala que las matemáticas están presentes en las actividades profesionales. En el ámbito 3 se destaca que un 51\% manifiesta estar completamente de acuerdo en que la matemática es una disciplina que ayuda a promover la creatividad en otras ramas. Finalmente, en el ámbito 4, los participantes le otorgan una alta valoración a las siguientes ideas: 1) un estudiante creativo es capaz de iniciar investigaciones (51\%) y sabe encontrar diferentes maneras de representar un concepto o problema $(63 \%)$, 2) un profesor creativo es transgresor con respecto a la enseñanza tradicional (51\%), tiene herramientas para estimular la creatividad (74\%) y sabe cómo valorar la creatividad en sus estudiantes (80\%) y 3) el quiebre disciplinar (60\%), un buen diseño de actividades (80\%) y la actitud del profesor (86\%) y del estudiante (57\%), son los elementos que tienen más impacto a la hora de promover la creatividad matemática en el aula.

Las respuestas a las afirmaciones presentadas en la tabla 1 se pueden profundizar a partir de los datos suministrados por los profesores participantes en las preguntas abiertas del instrumento, cuyo análisis permite reconocer, en primera instancia, las categorías y subcategorías presentadas en la figura 1, las que emergen de manera inductiva. Asimismo, tal como se ha señalado en el apartado anterior, se profundiza la información a través del análisis del discurso de los sujetos de investigación con base a los Criterios de Idoneidad (categorías deductivas).

Figura 1- La creatividad en el profesor de matemática

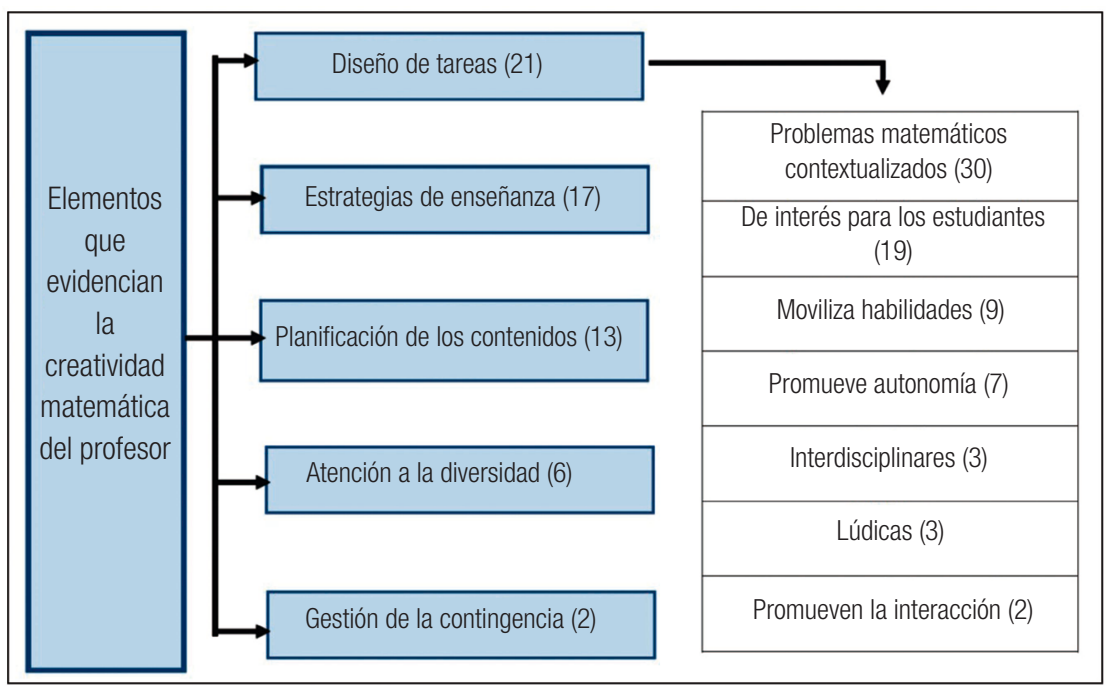

Fuente: elaboración propia. 
El cuestionario de preguntas abiertas consideró cinco cuestiones en relación a la creatividad en la labor del profesor de matemática. En la figura 1 se sintetiza el análisis de los datos recolectados en dos de ellas: 1) dónde se refleja la creatividad en tu ámbito profesional y 2) cuál/es característica/s consideras que debe tener una actividad matemática que promueva la creatividad. En las respuestas obtenidas en la primera pregunta se observa una alta frecuencia (21 participantes) al considerar el diseño de tareas como un elemento en el que se puede evidenciar la creatividad en la labor del profesor. El hallazgo de este elemento resulta interesante ya que, según las categorias de tipo deductivas propuestas en este estudio, en el proceso de diseño de tareas el profesor debiese considerar, a priori, los seis criterios de idoneidad didáctica. Lo mismo sucede con las categorías denominadas estrategias de enseñanza (con 17 participantes) y planificación de contenidos (con 13 participantes). Ahora bien, en el análisis de las respuestas, se levantan categorías que se relacionan con algunos de los CI, por ejemplo, la atención a la diversidad (idoneidad cognitiva) y la gestión de la contingencia (idoneidad interaccional), sin embargo, la frecuencia de cada una de ellas es más bien baja (6 y 2 participantes respectivamente).

A partir de la segunda pregunta se puede distinguir que la mayoría de los participantes (30 de un total de 35) coinciden en que los problemas matemáticos contextualizados son una característica de una actividad matemática que promueve la creatividad, lo que se relaciona con la idoneidad emocional, específicamente, con el componente intereses y necesidades y con el indicador proposición de situaciones que permitan valorar la utilidad de las matemáticas en la vida cotidiana y profesional. En segundo lugar, más del cincuenta por ciento de los participantes consideran que dichas actividades deben ser de interés para los estudiantes, lo que tiene relación con la idoneidad emocional, con el componente intereses y necesidades y con el indicador selección de tareas de interés para los alumnos. En esta misma línea, pese a que se reconocen menos registros en el discurso, también emergen como características la movilización de habilidades (relacionado con la idoneidad epistémica), promoción de autonomía (relacionado con la idoneidad interaccional), interdisciplinariedad (relacionado con la idoneidad ecológica), actividades lúdicas (relacionado con la idoneidad emocional) y la promoción de la interacción (relacionado con la idoneidad interaccional).

La tercera pregunta invitaba a los participantes a señalar un ejemplo de actividad que promueve la creatividad matemática y la cuarta estaba direccionada a lo que se podría hacer para promover la creatividad matemática en la enseñanza y aprendizaje de esta disciplina. En la tabla 2 y 3 se puede reconocer los tipos de actividades y acciones mencionadas presentando, a su vez, la frecuencia de aparición (número de personas que evidencia unidades de registros en cada categoría de tipo inductiva). 
Tabla 2- Tipos de actividades que promueven la creatividad matemática

\begin{tabular}{|c|c|}
\hline Tipo de actividad & Frecuencias de aparición \\
\hline Resolver problemas en contextos reales & 5 \\
\hline Crear problemas & 4 \\
\hline Crear juegos & 3 \\
\hline Construcciones geométricas & 2 \\
\hline Uso de tics & 2 \\
\hline Descubrir patrones & 2 \\
\hline Solicitar distintas propuestas para resolver un problema & 1 \\
\hline Creación de obras teatrales & 1 \\
\hline Diseñar herramientas & 1 \\
\hline Experimentos & 1 \\
\hline
\end{tabular}

Fuente: elaboración propia.

Tabla 3- Tipos de acciones que promueven la creatividad matemática en la enseñanza y aprendizaje de las matemáticas

\begin{tabular}{|c|c|}
\hline Tipo de acción & Frecuencia de aparición \\
\hline Presentación de problemas abiertos & 3 \\
\hline Capacitarse & 2 \\
\hline Actividades interdisciplinares & 4 \\
\hline Innovación en el aula & 4 \\
\hline Aprendizaje basada en proyectos & 3 \\
\hline Contextualizar la enseñanza & 10 \\
\hline Evaluar lo actitudinal & 1 \\
\hline Promover la interacción & 4 \\
\hline
\end{tabular}

Fuente: elaboración propia.

La segunda fase del análisis se relaciona, sobretodo, con la quinta pregunta del cuestionario que consistía en que los participantes presentasen un ejemplo de actividad matemática creativa y que tratasen de destacar lo creativo de ella. Un total de veintitrés participantes contestaron dicha pregunta, señalando que sus actividades fueron creativas porque consideran aspectos relacionados con los criterios de idoneidad: a) epistémica; b) mediacional; c) emocional; y d) ecológica.

En relación al CI epistémica, tres profesores consideran que se promueve la creatividad matemática si se trabajaban tareas que generan procesos matemáticos relevantes en los estudiantes, como la resolución de problemas, la deducción y la argumentación, lo que tiene estrecha relación con el componente denominado riqueza de procesos: 
Solicitar a los estudiantes ( $4^{\circ}$ básico) que aprendan a expresar inecuaciones en el contexto de resolución de problemas, a partir de las señales del tránsito. (participante A).

Un ejemplo de actividad matemática creativa, sería por ejemplo, que el alumno pudiera deducir reglas a partir de una actividad solicitada. El deducir reglas debe ser fomentado por la creatividad del alumno de encontrar estas reglas asociadas. (participante B).

Que los alumnos creen sus situaciones problemas y las expliquen y justifiquen. (participante C).

En relación al CI mediacional, tres profesores consideran que se promueve la creatividad matemática en la enseñanza si se realizan propuestas basadas en el uso de las TIC, cuyas evidencias se relacionan, específicamente, con el componente denominado recursos materiales:

Programa computacional con análisis estadístico de la población. (participante D).

Hice un proyecto para que estudiantes de tercer año medio enseñaran la parábola a través de videos, comics, entre otros. Lo creativo fue que utilizaron los videojuegos donde ellos vieron la parábola y conceptualizaron el concepto con el juego. (participante E).

El trabajo en probabilidades, el uso del geogebra y de simuladores de juego, incluyendo el obtener un número en forma aleatoria. El realizar presentaciones en prezi y la utilización de webquest en forma de apoyo a la autonomía del estudiante (el uso de tics para los estudiantes fue creativo, en la medida que entrega una herramienta que engrandece finalmente el aprendizaje de los estudiantes). (participante F).

En este mismo ámbito, ocho de los participantes consideraron que es el uso de material concreto que genera la creatividad matemática en los estudiantes:

Enseñar raíces y algoritmos con tangram, con alumnos de segundo medio al trabajar con operatoria básica de raíces (participante G).

Construir raíces cuadradas con regla y compás, luego de enseñar y aplicar matemática. (participante H).

Diseñar un juego matemático a partir de un objeto matemático. Fue creativo ya que fue necesario interiorizarse en la temática y crear un juego que funcione con el concepto. (participante I).

Un ejemplo es que el niño encuentre los caminos desde la plaza hasta su casa en un mapa. El alumno puede proponer distintas notas a partir de su creatividad (participante J).

Enseñar la diferencia del concepto de número y cifras por medio del juego al cambiar carteles y explicar el valor posicional que se genera al modificar el orden de cifras de un número específico. (participante K). 
Uso de la estrategia "rompecabezas" para la resolución de diversas temáticas estudiadas en clases en el área de matemática en segundo básico. (participante L).

Armar la red de un paralelepípedo y que los alumnos pintaran sus caras iguales (las del mismo tamaño) y que ellos dedujeran la fórmula a partir de esta actividad para calcular su área y a la vez comprender la fórmula. (participante $\mathrm{M}$ ).

Que el estudiante, en grupos, participe de un proyecto donde tengan que construir mobiliario que sea de utilidad para ellos considerando materiales reciclables y/o de bajo costo (presupuesto acotado). (participante N).

En relación al CI emocional, en particular al componente denominado intereses y necesidades (proposición de situaciones que permitan valorar la utilidad de las matemáticas en la vida cotidiana y profesional), tres profesores coincidieron en que la creatividad de los estudiantes es activada cuando se relaciona las matemáticas con situaciones contextualizadas:

Un ejemplo sería que para el estudio del teorema de Thales, se da a los estudiantes la tarea de medir un edificio mediante la indicación que tomen la medida de una persona de pie y la base de otra recostada a una distancia que consideren necesaria para estar en un ángulo de acuerdo a la persona que está en el piso. Sólo con la actividad realizada los estudiantes deben realizar todo lo necesario para el cálculo y lograr llegar a definir el teorema de Thales. (participante 0).

Determinar altura de un edificio, debemos sacar a los alumnos de su hábitat (sala de clases). Y dar la oportunidad de trabajar teorema de Thales, obteniendo información a través de datos que ellos van obteniendo. (participante P).

Un ejemplo de actividad matemática creativa, según mi percepción, es que los estudiantes midan la cancha de fútbol de su establecimiento, vean si se encuentra en las proporciones establecidas por la FIFA y vuelvan a trazar los límites si esta no se encuentra dentro de los parámetros establecidos. (participante Q).

En este mismo ámbito, respecto al descriptor Selección de tareas de interés para los alumnos, tres profesores declaran:

Dar un contenido y pedir al alumno que lo relacione con la rama o actividad que le parece interesante o se maneja. (participante U).

Considerar en primera instancia un problema cualquiera de un libro y en conjunto con los alumnos armar otro. Con datos cambiados, de acuerdo a los intereses de ellos en el momento, considerando sus conocimientos. (participante V).

En la dinámica diaria del aula que permite libre expresión a todos. (participante X). 
Finalmente, se puede observar que, para dos profesores, la creatividad en la enseñanza se relaciona con el CI ecológica, específicamente, las evidencias se relacionan con el componente conexiones intra e interdisciplinares, en este caso, hacen mención a relacionar las matemáticas con otras áreas de estudios:

Me gustaría estar en un salón muy amplio con muchos materiales a disposición de mis alumnos para que construyan una maqueta de un puente. Me acompañan el profesor de historia y el de física. El profesor de historia tendrá la misión de darles una visión histórica de la construcción de puentes, el de física hará su aporte siendo su guía en lo concerniente a la resistencia de materiales y yo les ayudaré con los cálculos... En equipos tendrán que elegir los materiales propicios para la construcción de un puente resistente que será sometido a prueba. (participante R).

Actividades generadas con otras áreas de la enseñanza. (participante S).

La tercera fase de análisis permite establecer dos ideas núcleo al contrastar los resultados obtenidos en las preguntas cerradas y abiertas del instrumento aplicado:

- Visión dual respecto a la creatividad matemática: en el apartado de preguntas cerradas se observa que un 54 por ciento de los participantes se manifiesta de acuerdo o totalmente de acuerdo en que la creatividad matemática es una capacidad innata. Asimismo, un porcentaje aún mayor (87\%) señala que esta se puede desarrollar y educar. Esta última afirmación se reafirma a partir de las categorías levantadas en el análisis de las preguntas abiertas, pues se destacan como elementos de la creatividad matemática del profesor procesos propios de la instrucción (planificación, diseño de tareas y estrategias de enseñanza).

- Características de procesos de enseñanza y aprendizaje que promueven la creatividad matemática:

1. Interdisciplinariedad: esta característica tiene relación con el CI ecológico, pues en él se establece la relación de las matemáticas con otras disciplinas. Es posible observar la presencia de este CI en las respuestas entregadas en los reactivos 7, 15 y 24 de las preguntas cerradas y en las categorías que se levantan a partir de las preguntas abiertas, donde el diseño de tareas interdisciplinares (como elemento que evidencia la creatividad matemática del profesor), las actividades interdisciplinares (como acciones que promueven la creatividad matemática) y las conexiones interdisciplinares (como ejemplos de una actividad que promueve la creatividad matemática) emergieron de las respuestas de los participantes.

2. Resolución de problemas con contextos reales: esta característica tiene relación con dos CI. En primer lugar, con el CI epistémico, pues en él se considera como componente la riqueza de procesos, que tiene relación con la consideración de procesos relevantes en la resolución de tareas, entre ellas, la resolución de problemas. En segundo lugar, se relaciona con el CI emocional, ya que éste 
contiene el componente intereses y necesidades, que considera la presentación de situaciones que permitan valorar las matemáticas en la vida cotidiana y profesional. Esto se puede evidenciar en el apartado de preguntas cerradas, donde se observa un alto porcentaje de profesores que valoran el buen diseño de actividades como elemento de impacto a la hora de promover la creatividad matemática dentro del aula (reactivo 25). Esto lo ratifican cuando responden las preguntas abiertas del cuestionario, pues la resolución de problemas en contextos reales, actividades en contexto o contextualización de la enseñanza fueron categorías que aparecieron con frecuencia en el discurso de los participantes.

3. Prácticas de enseñanza no tradicionales: esta característica se relaciona con el CI Mediacional, específicamente con el componente de recursos materiales, dado que los participantes manifiestan que la introducción de las TIC en el aula, son una alternativa para acabar con prácticas tradicionales de enseñanza. Esto se puede observar en el apartado de preguntas cerradas (reactivo 18). Asimismo, en el reactivo 23 se observa una alta valoración a la incorporación de nuevas tecnologías en el aula matemática como elemento que permite promover la creatividad matemática. En esta línea, en el análisis de las preguntas abiertas se levantó la categoría uso tics, la que en este estudio se interpretan como una alternativa de los profesores para lograr transgredir la enseñanza tradicional.

4. Interacción con otros: esta última característica se relaciona con el CI interaccional, donde se puede establecer conexión con dos de sus componentes, tanto con la interacción docente-discente, como con la interacción entre discentes. Las evidencias para esta característica se pueden observar en el análisis del reactivo 5, donde un 95 por ciento de los participantes manifiesta estar de acuerdo o totalmente de acuerdo en que la interacción con otras personas y/o diferentes puntos de vista es importante a la hora de desarrollar un trabajo creativo, como en el análisis de las preguntas abiertas, ya que el discurso de los profesores da énfasis al diseño de tareas que promueven la interacción como acción para promover la creatividad matemática en los alumnos y como elemento para reconocer la creatividad matemática del profesor.

\section{Conclusiones}

Guzmán (1995) planteaba que aún era un misterio la forma o los mecanismos que permiten promover la creatividad matemática, sin embargo, en este estudio los participantes evidencian consenso respecto a cuatro elementos que consideran clave para el desarrollo de la creatividad matemática, estos son, el trabajo interdisciplinar en el aula (CI ecológica), la resolución de problemas en contextos extramatemáticos (CI epistémica $y$ emocional), la introducción de TIC en el aula matemática como medida para transgredir las prácticas de enseñanza tradicionales (CI mediacional) y la interacción con otros (CI interaccional). Esta última puede relacionarse con otra de las competencias deseadas en la educación del siglo XXI, la colaboración.

Por otra parte, es posible reconocer que las dos visiones de creatividad matemática planteadas por Silver (1997) y Sriraman (2009) existen de manera dual en la concepción 
de los profesores participantes, es decir, estos consideran que la creatividad matemática puede ser una capacidad innata pero, en el caso que no se presente de esta manera, puede educarse o desarrollarse.

Como hemos señalado, Ervynck (1991) describe la creatividad matemática como etapas que se desarrollan a partir del planteamiento de un problema y su proceso de resolución, característica que han considerado los participantes del estudio. Ahora bien, Liljedahl y Sriraman (2006) profundizan esta idea señalando que a nivel escolar la creatividad matemática es el proceso que da como resultados soluciones inusuales (u originales) a un problema, afirmación que comparte la mayoría de los participantes (57\%), lo que se puede confirmar en las respuestas entregadas en el reactivo 8 del instrumento aplicado.

Consideramos que el presente trabajo contribuye a los estudios emergentes sobre el desarrollo conceptual en la formación de profesores de matemática, específicamente sobre creatividad matemática, proporcionando evidencia de la visión de los profesores participantes. A partir de sus respuestas se logra identificar que cuando estos argumentan sobre creatividad o actividad creativa, y analizamos los resultados de manera global (a través de los datos aportados por todos los participantes), es posible reconocer de manera implícita la presencia de todos los CI, fenómeno que coincide con lo observado en las investigaciones de Ramos (2006), Breda (2016), Seckel y Font (2020) y Morales y Font (2017). Estas evidencias permiten proyectar futuras investigaciones en las que se considere el diseño de un ciclo formativo para el desarrollo de la creatividad matemática en profesores o futuros profesores de matemática, así como el desarrollo de herramientas profesionales que les permita desarrollar y evaluar esta competencia en estudiantes del sistema escolar.

Respecto a las limitaciones del estudio, es importante destacar que los sujetos participantes se encuentran en la etapa final de su formación de máster en Educación Matemática y se desconoce si en su periodo de estudio conocieron el constructo de Idoneidad Didáctica, sin embargo, en el apartado de preguntas abiertas no hicieron referencia explícita a ninguno de los CI.

\section{Referencias}

BARQUERO, Berta et al. Promoviendo la creatividad matemática a través del diseño colaborativo de c-unidades. In: GONZÁLEZ, MARÍA TERESA et al. (Org.). Investigación en educación matemática XVIII. Salamanca: Seiem, 2014. p. 157-166.

BREDA, Adriana. Características del análisis didáctico realizado por profesores para justificar la mejora en la enseñanza de las matemáticas. Bolema (Rio Claro), en prensa.

BREDA, Adriana. Melhorias no ensino de matemática na concepção de professores que realizam o mestrado PROFMAT no Rio Grande do Sul: uma análise dos trabalhos de conclusão de curso. Porto Alegre: PUCRS, 2016. 326 f. Tesis (Doctorado en Educación en Ciencias y Matemática) - Pontifícia Universidade Católica do Rio Grande do Sul, Porto Alegre, 2016. 
BREDA, Adriana; FONT, Vicenç; LIMA, Valderez Marina do Rosario. A noção de idoneidade didática e seu uso na formação de professores de matemática. Jornal Internacional de Estudos em Educação Matemática, São Paulo, v. 8, n. 2, p. 4-41, 2015. DOl: 10.17921/2176-5634.2015v8n2p\%25p

BREDA, Adriana; FONT, Vicenç; PINO-FAN, Luis Roberto. Criterios valorativos y normativos en la didáctica de las matemáticas: el caso del constructo idoneidad didáctica. Bolema, Rio Claro, v. 32, n. 60, p. 255-278, 2018. DOl: 10.1590/1980-4415v32n60a13

BREDA, Adriana; LIMA, Valderez Marina do Rosario. Estudio de caso sobre el análisis didáctico realizado en un trabajo final de un máster para profesores de matemáticas en servicio. Redimat, Barcelona, v. 5, n. 1, p. 74-103, 2016. D0I: 10.4471/redimat.2016.1955

BREDA, Adriana; PINO-FAN, Luis Roberto; FONT, Vicenç. Meta didactic-mathematical knowledge of teachers: criteria for the reflection and assessment on teaching practice. Eurasia, London, v. 13, n. 6 , p. 1893-1918, 2017. DOI: 10.12973/eurasia.2017.01207a

CORFO; Fundación Chile. Preparando a Chile para una sociedad del conocimiento: hacia una coalición que impulse la educación STEAM. Santiago de Chile: Corfo, 2017. Disponible en: <https://fch.cl/wp-content/ uploads/2017/10/STEM_FCh_digital.compressed.pdf>. Acceso en: 08 my. 2018.

ERVYNCK, Gontran. Mathematical Creativity. In: TALL, David (Ed.). Advanced mathematical thinking. Netherlands: Springer, 1991. p. 42-53.

FONT, Vicenç; PLANAS, Nuria; GODINO, JUAN DÍAZ. Modelo para el análisis didáctico en educación matemática. Infancia y Aprendizaje, London, v. 33, n. 1, p. 89-105, 2010.

GUZMÁN, Miguel. Para pensar mejor: desarrollo de la creatividad a través de los procesos matemáticos. Madrid: Pirámide, 1995.

HADAMARD Jacques. An essay on the psychology of invention in the mathematical field. New Jersey: Princeton University Press, 1945.

HAYLOCK, Derek. A framework for assessing mathematical creativity in school children. Educational Studies in Mathematics, Netherlands, v.18, n.1, p. 59-71, 1987.

KAUFMAN, James; BEGHETTO, Ronald. Exploring "mini-c:" Creativity across cultures. In: DeHaan, Robert; Narayan, K. M. Venkat (Ed.). Education for Innovation in India, China and America. Netherlands: Sense, 2008. p. 165-180.

LILJEDAHL, Peter. Illumination: an affective experience? The International Journal on Mathematics Education, Berlín, v. 45, n. 2, p. 253-265, 2013.

LILJEDAHL, Peter; SRIRAMAN, Bharath. Musing on mathematical creativity. For the Learning of Mathematics, Edmonton, v. 26, n. 1, p. 20-23, 2006.

LOGAN, Lilian; LOGAN, Virgil; GARCÍA, Alicia Ramón. Estrategias para una enseñanza creativa. Barcelona: Oikos-tau, 1980. 
MALLART, Alberto; DEULOFEU, Jordi. Estudio de indicadores de creatividad matemática en la resolución de problemas. Revista Latinoamericana de Investigación en Matemática Educativa, Ciudad de México, v. 20, n. 2, p. 193-222, 2017.

MANN, Eric. Creativity: the essence of mathematics. Journal for the Education of the Gifted, New York, v. 30, p. 236-230, 2006.

MARÍN, Ricardo; DE LA TORRE, Saturnino. Manual de la creatividad. Barcelona: Vicens Vives, 1991.

MORALES, Yuri; FONT, Vicenç. Análisis de la reflexión presente en las crónicas de estudiantes en formación inicial en educación matemática durante su periodo de práctica profesional. Acta Scientiae, Canoas, v. 19, n. 1, p. 122-137, 2017.

MUÑIZ, José. Teoría clásica de los tests. Madrid: Pirámide, 2017.

RAMOS, Ana Beatriz. Objetos personales, matemáticos y didácticos, del profesorado y cambios institucionales: el caso de la contextualización de las funciones en una facultad de ciencias económicas y sociales. Barcelona: UB, 2006. 378 p. Tesis (Doctorado) - Universitat de Barcelona, Barcelona, 2006.

REYES, Manuel. Las estrategias creativas como factor de cambio en la actitud del docente para la enseñanza de la matemática. Sapiens, Caracas, v. 4, n. 2, p.71-94, 2003.

SÁNCHEZ, Alicia; FONT, Vicenç. Reflexión sobre los futuros profesores de matemáticas y fomento de la creatividad en sus alumnos. In: CONTRERAS, José Miguel et al. (Ed.). Actas del segundo congreso international virtual sobre el enfoque ontosemiótico del conocimiento y la instrucción matemáticos. Granada: [s. n.], 2017. Disponible en: <enfoqueontosemiotico.ugr.es/civeos.html>. Acceso en: 09 my. 2018.

SECKEL, María José; FONT, Vicenç. Competencia reflexiva en formadores del profesorado de matemática. Magis, Bogotá, v. 12, n. 25, p. 127-144, 2020. D0I: 10.11144/Javeriana.m12-25.crfp

SILVER, Edward. Fostering creativity through instruction rich in mathematical problem solving and problem posing. ZDM Mathematics Education, Berlín, v. 29, n. 3, p. 75-80, 1997.

SRIRAMAN, Bharath. Are giftedness and creativity synonyms in mathematics? Prufrock Journal, EE.UU, v. 17, n. 1, p. 20-36, 2005.

SRIRAMAN, Bharath. The characteristics of mathematical creativity. ZDM Mathematics Education, Berlín, v. 41, n. 1-2, p. 13-27, 2009.

THOMPSON, Alba. Teacher's beliefs and conceptions: a synthesis of the research. In: GROUWS, Douglas A. (Ed.). Handbook of research on mathematics teaching and learning. New York: Macmillan, 1992. p. 127-146.

VIOLANT, Verónica. Indicadores clásicos en la evaluación de la creatividad. In: DE LA TORRE, Saturnino; VIOLANT, Verónica (Org.). Comprender y evaluar la creatividad. Archidona: Aljibe, 2006. p. 169-179. 
María José Seckel es doctora en Formación del Profesorado: práctica educativa y comunicación por la Universitat de Barcelona (España). Profesora del departamento de Formación inicial escolar de la Universidad Católica del Maule (Chile).

Adriana Breda es doctora en Educación en Ciencias y Matemática por la Pontifícia Universidade Católica do Rio Grande do Sul (Brasil) y postdoctoranda en la Universitat de Barcelona (España).

Alicia Sánchez es máster de Profesor de Secundaria de Matemáticas por la Universitat de Barcelona (España). Estudiante del programa de doctorado en Didáctica de las Ciencias, las Lenguas, las Artes y las Humanidades de la Universitat de Barcelona (España).

Vicenç Font es doctor en Filosofía y Ciencias de la Educación por la Universitat de Barcelona (España). Profesor del departamento de Educación Lingüística y Literaria y de Didáctica de las CCEE y de la Matemática de la Universitat de Barcelona (España). 